\title{
Adaptive Control Algorithm of the Synchronous Generator
}

\author{
Victor Shevchenko, Viacheslav Pshikhopov, Mikhail Medvedev \\ Southern Federal University, 347922, Russia, Rostov Region, Taganrog, Shevchenko st., 2.
}

\begin{abstract}
The article discusses the the problem of controlling a synchronous generator, namely, maintaining the stability of the control object in the conditions of occurrence of noise and disturbances in the regulatory process. The model of a synchronous generator is represented by a system of differential equations of Park-Gorev, where state variables are computed relative to synchronously rotating $d$, q-axis. Management of synchronous generator is proposed to organize on the basis of the position-path control using algorithms to adapt with the reference model. Basic control law directed on the stabilizing indicators the frequency generated by the current and the required power level, which is achieved by controlling the mechanical torque on the shaft of the turbine and the value of the excitation voltage of the synchronous generator. Modification of the classic adaptation algorithm using the reference model, allowing to minimize the error of the reference regulation and the model under investigation within the prescribed limits, produced by means of the introduction of additional variables controller adaptation in the model. Carried out the mathematical modeling of control provided influence on the studied model of continuous nonlinear and unmeasured the disturbance. Simulation results confirm the high level accuracy of tracking and adaptation investigated model with respect to the reference, and the present value of the loop error depends on parameters performance of regulator.
\end{abstract}

\section{Introduction}

Development of the control system for the synchronous generator (SG), providing required degree of quality generated power and operation parameters of the generator, is a actual task in connection with concept of universal electrification, use energy saving and energy-efficient technologies, increase safe and sustainable functioning of the power grid. Special problem in the task of control system synthesis for synchronous generator is construction of the control, able to adapt to arising perturbations which appeared in the regulation process. [1]. Accuracy and quality of system must be invariably independently of changing object parameters. Formation a controller that can able adapt to external perturbations possibly with use adaptive control systems. Adaptation algorithms with reference model is used when object model is known and unknown parameters, but in classic execution this system unable adequately react on arising perturbation during operation [2]. This problem can be decided by introduction in object model additional parameters [3] which allow to adapt error of regulation in reference model and investigated model in set limits.

Basic control law for synchronous generator is constructed on the principles of position-trajectory control This control successfully used in robotic mobile systems [3-10], such as control systems of autonomous marine devices [3-5] and aeronautic complex [7, 10], and for constructing regulator of stationary systems [1 - 2], such as control systems of traction electric motor [8], DC motor, synchronous generator [2], etc.

\section{Synthesis of adaptive control}

Mathematical model of synchronous generator is presented system differential equations by Park-Gorev. State variables in this model is currents id, iF, iq (instantaneous value), deviation of angle $\delta$ and angular velocity $\omega$. Model of synchronous machine, operating at the system of unlimited capacity across power line, can be presented follows: [2]:

$$
\begin{gathered}
\frac{d \delta}{d \tau}=\omega-1, \\
\frac{d \omega}{d \tau}=-\frac{L_{d}}{3 \tau_{J}} i_{q} i_{d}-\frac{k M_{F}}{3 \tau_{J}} i_{q} i_{F}+\frac{L_{q}}{3 \tau_{J}} i_{d} i_{q}-\frac{D}{\tau_{J}}(\omega-1)+\frac{M_{M X}}{\tau_{J}}, \\
\frac{d i_{d}}{d \tau}=\frac{-L_{F}\left(r_{s}+r_{e}\right)}{L} i_{d}-\omega \frac{L_{F}\left(L_{q}+L_{e}\right)}{L} i_{q}+\frac{k M_{F} r_{F}}{L} i_{F}-\frac{k M_{F}}{L} u_{F}+ \\
+\frac{L_{F} U_{\infty} \sqrt{3}}{L} \sin (\delta) \\
\frac{d i_{F}}{d \tau}=\frac{k M_{F}\left(r_{s}+r_{e}\right)}{L} i_{d}+\frac{-\left(L_{d}+L_{e}\right)}{L} r_{F} i_{F}+\omega \frac{k M_{F}\left(L_{q}+L_{e}\right)}{L} i_{q}+\frac{\left(L_{d}+L_{e}\right)}{L} u_{F}- \\
-\frac{k M_{F} U_{\infty} \sqrt{3} \sin (\delta)}{L} \\
\frac{d i_{q}}{d \tau}=\frac{\left(L_{d}+L_{e}\right)}{\left(L_{q}+L_{e}\right)} \omega i_{d}+\frac{k M_{F}}{\left(L_{q}+L_{e}\right)} \omega i_{F}-\frac{\left(r_{s}+r_{e}\right)}{\left(L_{q}+L_{e}\right)} i_{q}-\frac{U_{\infty} \sqrt{3}}{\left(L_{q}+L_{e}\right)} \cos (\delta)
\end{gathered} .
$$

where $L=L_{F}\left(L_{d}+L_{e}\right)-k M_{F}^{2} ; L_{d}, L_{q}, L_{F}$ - own inductance of stator windings by longitudinal $d$ and transverse $q$ axis and inductance of rotor winding, $M_{F}$ - mutual inductance of starter and rotor windings, $r_{s}, r_{F}, r_{e}$ - active resistance of the stator windings, the excitation winding of the rotor and transmission lines, $U_{\infty}, u_{F}$ - voltage of infinite bus power 
and the excitation winding of the rotor, $k$ - Park's constant, $\tau_{J}$ - mechanical time constant. All model parameters, including current, voltage and time is given in relative units.

This mathematical model of the reference and test patterns is represented by the system of equations (1), in the reference model for the state variables used, the index m.

Will make the control synthesis for the reference model, stabilizing the current frequency and power of the generator. To control these options control the mechanical torque on the turbine shaft and the excitation voltage. In accordance with the synthesis method of position-trajectory control we introduce the error control in the form:

$$
\psi_{\text {эm }}=A_{1} \cdot y_{m}-A_{2}
$$

$$
\text { where } y_{m}=\left[\begin{array}{c}
\omega_{m} \\
P_{g m}
\end{array}\right] ; T=\left[\begin{array}{c}
T_{w} \\
T_{p}
\end{array}\right] ; A_{1}=\left[\begin{array}{c}
A_{1 w} \\
A_{1 p}
\end{array}\right] ; A_{2}=\left[\begin{array}{c}
A_{2 w} \\
A_{2 p}
\end{array}\right] ;
$$

To ensure the asymptotic convergence of the estimation, we require that the error (2) subordinated to the solution of the equation:

$$
\dot{\psi}_{\text {эm }}+T \cdot \psi_{\text {эm }}=0
$$

The first derivative error (2) will be as follows:

$$
\dot{\psi}_{\text {эm }}=A_{1} \cdot \dot{y}_{m}
$$

Thus, equation (3) with (2) and (4) we write as:

$$
A_{1} \cdot \dot{y}_{m}+T \cdot A_{1} \cdot y_{m}-T \cdot A_{2}=0
$$

In scalar form equation (5):

$$
\left\{\begin{array}{l}
A_{1 w} \cdot \dot{\omega}_{m}+T_{w} \cdot A_{1 w} \cdot \omega_{m}-T_{w} \cdot A_{2 w}=0 \\
A_{1 p} \cdot \dot{P}_{g m}+T_{w} \cdot A_{1 p} \cdot P_{g m}-T_{w} \cdot A_{2 p}=0
\end{array}\right.
$$

For ease of presentation and solution of the equations we introduce some reduction resulting from the system (1):

$$
\begin{gathered}
\dot{\omega}_{m}=f_{w_{-} m}+M_{m x_{-} m} / \tau_{j} \\
\dot{P}_{g m}=6 \cdot \sqrt{3} \cdot R_{n} \cdot\left(f_{12_{-} m}+\frac{k M_{F}}{L} U_{F_{-} m} \cdot \sin \left(\delta_{m}\right)\right) \\
f_{12_{-} m}=f_{1 m}-f_{2 m} \\
f_{1 m}=f_{i q m} \cdot \cos \left(\delta_{m}\right)-f_{i d m} \cdot \sin \left(\delta_{m}\right) \\
f_{2 m}=\left(i_{q m} \cdot \sin \left(\delta_{m}\right)+i_{d m} \cdot \cos \left(\delta_{m}\right)\right) \cdot\left(\omega_{m}-1\right) \\
-\frac{\left(L_{d}+L_{e}\right)}{\left(L_{q}+L_{e}\right)} \omega_{m} i_{d m}+\frac{k M_{F}}{\left(L_{q}+L_{e}\right)} \omega i_{F m}-\frac{\left(r_{s}+r_{e}\right)}{\left(L_{q}+L_{e}\right)} i_{q m}- \\
\cos \left(\delta_{m}\right) \\
f_{i d m}=\frac{-L_{F}\left(r_{s}+r_{e}\right)}{L} i_{d m}-\omega_{m} \frac{L_{F}\left(L_{q}+L_{e}\right)}{L} i_{q m}+ \\
+\frac{k M_{F} r_{F}}{L} i_{F m}+\frac{L_{F} U_{\infty} \sqrt{3}}{L} \sin \left(\delta_{m}\right)
\end{gathered}
$$

Substituting expressions (7) - (13) in (7) we obtain the equations of the controllers to the reference model:

$$
\left\{\begin{array}{l}
M_{m x_{-} m}=\left(\frac{-T_{w} \cdot A_{1 w} \cdot \omega_{m}+T_{w} \cdot A_{2 w}}{A_{1 w}}-f_{w_{-} m}\right) \cdot \tau_{j} \\
U_{F_{-} m}=\left(\frac{\left(-T_{w} \cdot A_{1 p} \cdot P_{g m}+T_{w} \cdot A_{2 p}\right)}{A_{1 p} \cdot 6 \cdot \sqrt{3} \cdot R_{n}}-f_{12_{-} m}\right) \cdot L /\left(k M_{F} \cdot \sin \left(\delta_{m}\right)\right)
\end{array}\right.
$$

Consider the synthesis of adaptive control for the investigated model, the control objective which is defined as follows:

$$
\psi=A_{1} \cdot y-A_{2}
$$

where $y=\left[\begin{array}{l}\omega \\ P_{g}\end{array}\right]$.

We introduce the additional variables of the adaptive controller $\mathrm{z}$ :

$$
\dot{z}=\left[\begin{array}{c}
\dot{z}_{1} \\
\dot{z}_{2}
\end{array}\right]=\left[\begin{array}{l}
\psi_{1}-\psi_{1 э m} \\
\psi_{2}-\psi_{2 э m}
\end{array}\right]
$$

Error control is specified as:

$$
e=\psi-\psi_{\text {эm }}+B \cdot z
$$

where $B=\left[\begin{array}{l}B_{1} \\ B_{2}\end{array}\right]$;

Further, similar to the procedure for the synthesis of the reference model will require the following equality:

$$
\dot{e}+T \cdot e=0
$$

where $e=\left[\begin{array}{l}e_{1} \\ e_{2}\end{array}\right]=\left[\begin{array}{l}\psi_{1}-\psi_{l э m}+B_{1} \cdot z_{1} \\ \psi_{2}-\psi_{2 э m}+B_{2} \cdot z_{2}\end{array}\right]$

Define the first derivative of the control error of (17) for the regulator's synthesis with considering the equations (2), (4), (15), (16)

$$
\begin{aligned}
& \dot{e}_{1}=A_{1 p} \dot{P}_{g}-A_{1 p} \dot{P}_{g m}+B_{1}\left(A_{l_{p}} P_{g}-A_{l p} P_{g m}\right) \\
& \dot{e}_{2}=A_{l p} \dot{P}_{g}-A_{l p} \dot{P}_{g m}+B_{l}\left(A_{l_{p}} P_{g}-A_{l p} P_{g m}\right)
\end{aligned}
$$

Solving equations (18) with respect to the mechanical torque on the shaft and the excitation voltage, obtain the following expression for the adaptive controller with reference model:

$$
\begin{gathered}
M_{m x}=\left(\frac{k_{w}}{A_{1 w}}-f_{w}\right) / \tau_{j} \\
U_{F}=\left(\frac{k_{p}}{6 \sqrt{3} \cdot A_{1 p}}-f_{12}\right) \cdot \frac{L}{k \cdot M_{F} \sin (\delta)}
\end{gathered}
$$

In equations (19) and (20) used the following abbreviations:

$$
\begin{aligned}
& k_{w}=A_{1 w} \cdot \dot{\omega}_{m}-B_{1} \cdot A_{1 w} \cdot \omega+B_{1} \cdot A_{1 w} \cdot \omega_{m}-T_{1} \cdot A_{1 w} \cdot \omega+, \\
& +T_{1} \cdot A_{1 w} \cdot \omega_{m}-T_{1} \cdot B_{1} \cdot z_{1} \\
& k p=A_{1 p} \cdot \dot{P}_{g m}-B_{2} \cdot A_{1 p} \cdot P_{g}+B_{2} \cdot A_{1 p} \cdot P_{g m}-T_{1} \cdot A_{1 p} \cdot P_{g}+ \\
& +T_{1} \cdot A_{1 p} \cdot P_{g m}-T_{1} \cdot B_{2} \cdot z_{2}
\end{aligned}
$$

as well as reduction as in (7)-(13). 
In the General case, adaptation of the control system to respond to perturbations is due to the automatic tuning of parameters $B_{1}$ and $B_{2}$.

\section{Modeling system}

Fig. 1 presents the structure of an adaptive control system, which shows that the control system of a synchronous generator is constructed as a servo outputs for the reference model system. In the linear approximation adaptation to perturbations provides astatism first order. Management is built on the basis of several control loops: reference model's loop, the base management loop and adaptation loop. This structure allows you to adjust adaptation parameters B1 and B2 regardless of the settings of the base regulator.

The result of this simulation system are presented in figures 2 to 5, where solid line denotes the parameters of the model, and the dashed line indicates the parameters of the reference model.

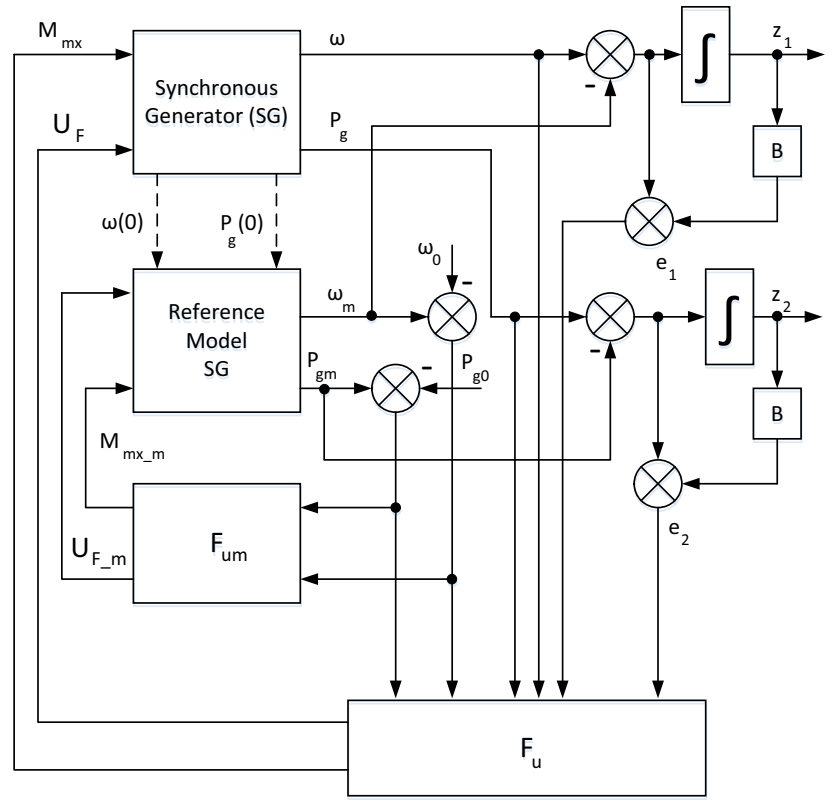

Figure 1. The structure of the adaptive control system of a SG

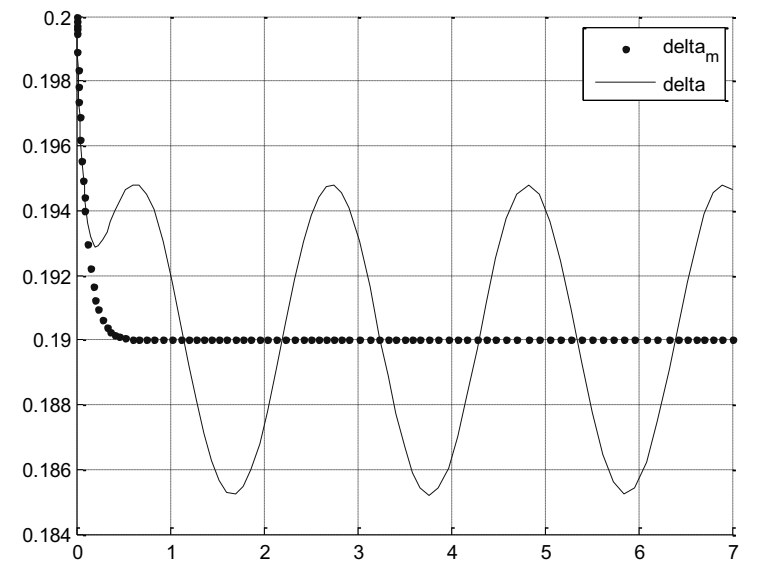

Figure 2. Angle $\delta$.

The simulation was performed using the following system parameters: $\mathrm{L}_{\mathrm{d}}=0.973 ; \mathrm{t}_{\mathrm{j}}=10 ; \mathrm{k}=1 ; \mathrm{M}_{\mathrm{f}}=0.923 ; \mathrm{L}_{\mathrm{q}}=0.55$; $\mathrm{D}=0 ; \mathrm{L}_{\mathrm{f}}=1.088 ; \mathrm{L}=1 ; \mathrm{L}_{\mathrm{e}}=0.997 ; \mathrm{r}_{\mathrm{s}}=0.005 ; \mathrm{r}_{\mathrm{e}}=0.005 ; \mathrm{r}_{\mathrm{f}}=0.14$; $\mathrm{U}_{\infty}=1 ; \mathrm{R}_{\mathrm{n}}=300 ; \mathrm{A}_{1 \mathrm{w}}=1 ; \mathrm{A}_{1 \mathrm{p}}=1 ; \mathrm{T}_{1 \mathrm{w}}=10 ; \mathrm{T}_{1 \mathrm{p}}=1 ; \mathrm{A}_{2 \mathrm{w}}=1 ; \mathrm{A}_{2 \mathrm{p}}=1$.
The disturbance acting on the reference model is zero, the perturbation value acting on the rotational speed and power $5 \cdot \sin (3 t)$.

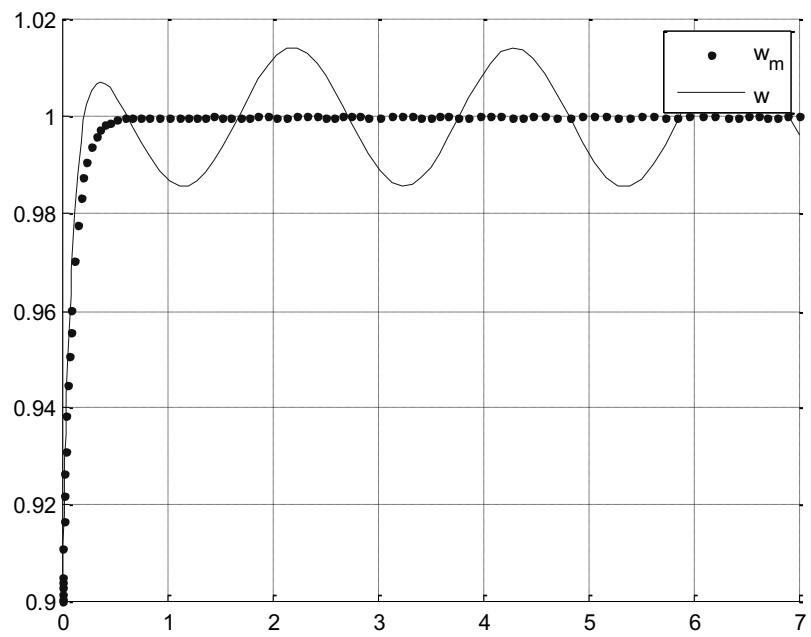

Figure 3. Shaft speed

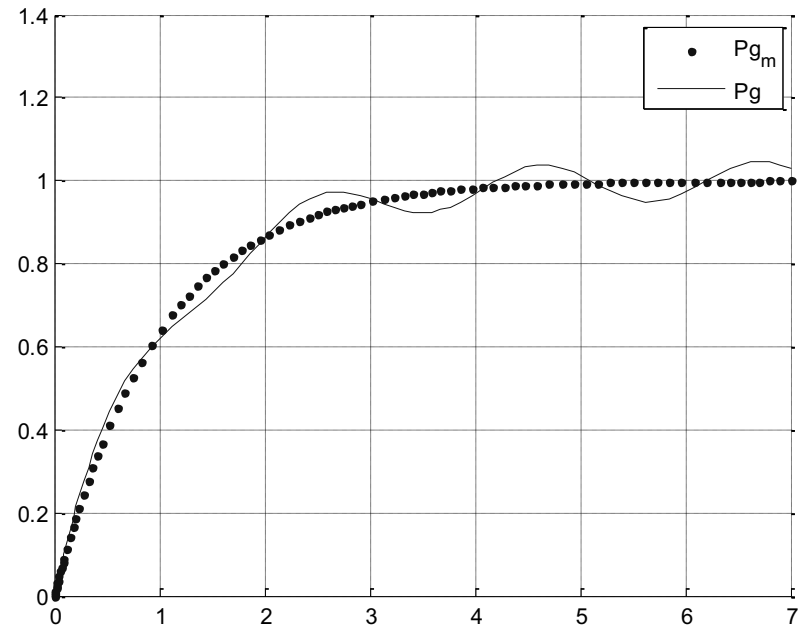

Figure 4. Generated power.

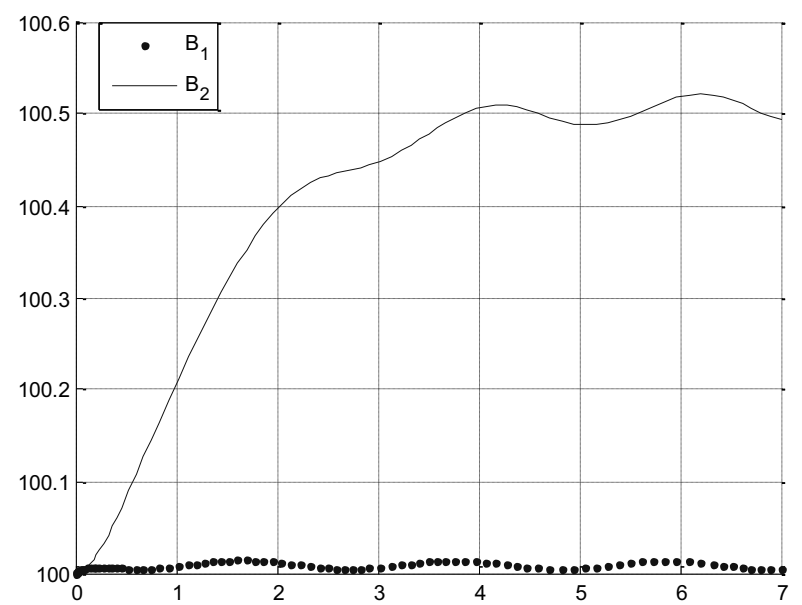

Figure 5. The coefficient of adaptation.

May be concluded from the results of simulation that the adaptive system with high precision monitors a reference model and allows you destroy the arising perturbations in the system under study and deviation values of the variables of 
the model in relation to the reference model depends on the speed controller parameters.

\section{Acknowledgement}

The work was supported by SFU (grant № 213.01-07/2014-01 "Theory and methods of energy saving control of distributed generation systems, transportation and consumption of electricity"). The reported study was funded by RFBR according to the research project № 16-08-00013 A.

\section{References}

1. Shevchenko, V.A., Pshikhopov, V.K., Medvedev, M.Y. Multilevel control system of the power grid // Advanced Materials Research, Volume 1049-1050, 2014, Pages 736-742.

2. Shevchenko, V.A. Development and research of system of automatic control by the synchronous generator // Proceedings of the 26th International Conference on Efficiency, Cost, Optimization, Simulation and Environmental Impact of Energy Systems, ECOS 2013.

3. Pshikhopov, V.K., Fedotov, A.A., Medvedev, M.Yu., Medvedeva, T.N., Gurenko, B.V. Position-trajectory system of direct adaptive control marine autonomous vehicles // 2014 the 4th International Workshop on Computer Science and Engineering - Summer, WCSE 2014.
4. Pshikhopov V.K., Medvedev M.Y., Gurenko B.V. Homing and docking autopilot design for autonomous underwater vehicle // Applied Mechanics and Materials. 2014. T. 490-491. C. 700-707.

5. Pshikhopov V.Kh., Medvedev M.Yu., Gaiduk A.R., Gurenko B.V. Control system design for autonomous underwater vehicle // Proceedings - 2013 IEEE Latin American Robotics Symposium, LARS 2013 2013. C. 77-82.

6. Medvedev M.Yu., Pshikhopov V.Kh. Design of robust control for block nonlinear systems by lyapunov functions method // Advanced Materials Research, Volume 1049-1050, 2014, Pages 1048-105.

7. Pshikhopov, V., Medvedev, M., Krukhmalev, V., Fedorenko, R., Gurenko, B. Method of docking for stratospheric airships of multibody transportation system // SAE Technical Papers, Volume 2014-September, Issue September, 16 September 2014.

8. Pshikhopov, V., Medvedev, M., Gaiduk, A. Control method for vehicles on base of natural energy recovery // Applied Mechanics and Materials, Volume 670-671, 2014, Pages 1330-1336

9. Neydorf, R., Krukhmalev, V., Kudinov, N., Pshikhopov, V. Methods of statistical processing of meteorological data for the tasks of trajectory planning of MAAT feeders // SAE Technical Papers, Volume 7, 2013.

10. Pshikhopov, V., Medvedev, M., Kostjukov, V., Fedorenko, R., Gurenko, B., Krukhmalev, V. Airship autopilot design // SAE Technical Papers, 2011. 